\title{
Efferent Protection from Acoustic Injury Is Mediated via $\alpha 9$ Nicotinic Acetylcholine Receptors on Outer Hair Cells
}

\author{
Stéphane F. Maison, ${ }^{1}$ Anne E. Luebke, ${ }^{2}$ M. Charles Liberman, ${ }^{1}$ and Jian Zuo ${ }^{3}$ \\ ${ }^{1}$ Department of Otology and Laryngology, Harvard Medical School and Eaton-Peabody Laboratory, Massachusetts Eye \\ and Ear Infirmary, Boston, Massachusetts 02114, ${ }^{2}$ Department of Otolaryngology and Neuroscience Program, University \\ of Miami School of Medicine, Miami, Florida 33136, and 'Department of Developmental Neurobiology, St. Jude Children's \\ Research Hospital, Memphis, Tennessee 38105
}

Exposure to intense sound can damage the mechanosensors of the inner ear and their afferent innervation. These neurosensory elements are innervated by a sound-activated feedback pathway, the olivocochlear efferent system. One major component of this system is cholinergic, and known cholinergic effects are mediated by the $\alpha 9 / \alpha 10$ nicotinic acetylcholine receptor (nAChR) complex. Here, we show that overexpression of $\alpha 9$ $\mathrm{nAChR}$ in the outer hair cells of bacterial artificial chromosome transgenic mice significantly reduces acoustic injury from ex-

The $\alpha 9$ nicotinic acetylcholine receptor (nAChR) is a subunit of a receptor family with 17 known members. As a group, they are widely and differentially expressed in the CNS and PNS (Jones et al., 1999). In vitro, these subunits form pentameric complexes surrounding a nonspecific cation channel (Cordero-Erausquin et al., 2000). The functional roles of these subunits range from mediating fast synaptic transmission in the autonomic nervous system to transducing neuroprotective and antinociceptive effects in vivo and in vitro (Jones et al., 1999).

Expression of the $\alpha 9 \mathrm{nAChR}$ is restricted to the anterior pituitary, the nasal epithelium, and the inner hair cells (IHCs) and outer hair cells (OHCs), the mechanosensors of the inner ear (Elgoyhen et al., 1994; Zuo et al., 1999). In the cochlea, the $\alpha 9$ subunit may act in concert with $\alpha 10$, the newest member of the nAChR family (Elgoyhen et al., 2001). However, the $\alpha 9$ subunit, per se, is essential for the best-studied cholinergic effect on cochlear function: the suppression of cochlear responses normally induced by activation of the cholinergic efferent system known as the olivocochlear (OC) pathway (Vetter et al., 1999).

In hair cells, $\alpha 9 / \alpha 10$ complexes are functionally coupled to $\mathrm{K}_{\mathrm{Ca}}$ channels; thus, ligand-gated $\mathrm{Ca}^{2+}$ entry through the nAChR (Fuchs and Murrow, 1992) is coupled to $\mathrm{K}^{+}$efflux and intracellular hyperpolarization (Housley and Ashmore, 1991). This hy-

Received Aug. 22, 2002; revised Sept. 24, 2002; accepted Sept. 27, 2002.

This work was supported by National Institutes of Health (NIH) Grants R01 DC-0188, R01 DC-03086, R01 EY12950, R21 DC 05168, and R03 DC-04761, NIH Cancer Center Grant CA-21765, Chiles Endowment Biomedical Research Program of the Florida Department of Health Grant BM028, March of Dimes Birth Defects Foundation Grant 5-FY98-0725, the American Lebanese Syrian Associated Charities, and a Long-Term Fellowship of the Human Frontier Science Program Organization (S.F.M.). We thank J. He, A. Lowrey, J. Treadaway, M. Valentine, and V. Valentine for assistance; Dr. T. Hasson (University of California, San Diego, CA) for providing the Myosin VI antibody; and Dr. J. J. Guinan Jr. for comments on this manuscript.

Correspondence should be addressed to Dr. M. Charles Liberman, EatonPeabody Laboratory, Massachusetts Eye and Ear Infirmary, 243 Charles Street, Boston, MA 02114-3096. E-mail: mcl@epl.meei.harvard.edu.

Copyright (C) 2002 Society for Neuroscience $0270-6474 / 02 / 2210838-09 \$ 15.00 / 0$ posures causing either temporary or permanent damage, without changing pre-exposure cochlear sensitivity to low- or moderate-level sound. These data demonstrate that efferent protection is mediated via the $\alpha 9 \mathrm{nAChR}$ in the outer hair cells and provide direct evidence for a protective role, in vivo, of a member of the nAChR family.

Key words: olivocochlear; nicotinic; cholinergic; noiseinduced hearing loss; transgenic mouse; cochlea

perpolarization of $\mathrm{OHCs}$, in turn, affects their electromotile responses (Dallos, 1992), decreasing cochlear mechanical responses to low-level sounds (Dolan and Nuttall, 1988; Murugasu and Russell, 1996) and elevating auditory thresholds (Wiederhold and Kiang, 1969).

The functional significance of this efferent feedback system remains controversial; however, a role in protection from acoustic injury has been proposed. In anesthetized animals, electrical stimulation of the efferent pathway reduces temporary hearing loss from simultaneous acoustic overexposure (Rajan, 1988; Reiter and Liberman, 1995); chronic surgical de-efferentation renders awake animals more vulnerable to permanent hearing loss from high-level noise (Kujawa and Liberman, 1997). Although an efferent role in protection is well established, the complexity of the OC system has hampered understanding of the mechanisms underlying this effect. At the neuroanatomical level, the efferent pathway consists of two subsystems (see Fig. 1): a medial component projecting primarily to $\mathrm{OHCs}$ and a lateral component primarily innervating afferent terminals on IHCs (Warr et al., 1986). At the cytochemical level, there is evidence for both GABAergic and cholinergic transmission in both medial and lateral systems (Eybalin, 1993) and evidence for dopaminergic and peptidergic transmission in the latter (Eybalin, 1993). To directly assess the role of the $\alpha 9 \mathrm{nAChR}$ in efferent-mediated protection from acoustic injury, we studied a transgenic ( $\mathrm{Tg}$ ) mouse line in which $\alpha 9$ is overexpressed. This report documents (1) the overexpression at the protein level, (2) the localization to OHCs via a green fluorescent protein (GFP) reporter, (3) the lack of effect of overexpression on baseline cochlear sensitivity, (4) the enhancement of electrically evoked OC effects on the cochlea, and (5) the resultant enhancement of resistance to both temporary and permanent acoustic injury.

\section{MATERIALS AND METHODS}

Experimental procedures. FVB/NJ mice were obtained from The Jackson Laboratory (Bar Harbor, ME). Transgenic lines were created (see be- 
low), maintained as heterozygotes in the original FVB/NJ strain background, and genotyped in the Zuo laboratory in Memphis [an extensive description of the methods is described by Zuo et al. (1999)]. Transgenic mice (both homozygous and heterozygous) and their wild-type littermates were shipped to Miami and Boston, where investigators were blinded to the genotype until all data acquisition was complete. For all physiological experiments, including baseline testing auditory sensitivity and magnitude of OC suppression, as well as in acoustic overexposure experiments, mice ranged in age from 10 to 12 weeks. Mice used for Western blot ranged in age from 4 to 12 weeks.

Transgenic construct and genotyping. Two homologous fragments that contain the coding portion and the $3^{\prime}$ untranslated region of exon 5 were used for modification of the transgene. An internal ribosome entry site/GFP cassette $(1.3 \mathrm{~kb}$ ) was inserted after the stop codon (see Fig. $2 \mathrm{~A}$ ) (Zuo et al., 1999). A second transgenic line was created with an identical construct except that exon 4 (E4) of the $\alpha 9$ nAChR gene was deleted, as in the $\alpha 9$ knock-out strategy (Vetter et al., 1999). This was achieved with two homologous fragments flanking the E4. The previously modified bacterial artificial chromosome (BAC), $\mathrm{mK}$, was further modified for such a deletion. The resulting BAC DNA, mK9E4, was used for transgenic injection as described previously. Mice were genotyped using both PCR and interphase fluorescence in situ hybridization (FISH) assays (see Fig. 2B) (Zuo et al., 1999). For the interphase FISH analysis, the ends $(\sim 0.5-1 \mathrm{~cm})$ of tails were removed from mice that were between the ages of 1 week and 9 months. The tail fragments were immersed in complete tissue culture medium (Roswell Park Memorial Institute 1640 ) at $4^{\circ} \mathrm{C}$ for up to $3 \mathrm{~d}$. The tail fragments were minced and then enzymatically disaggregated by collagenase ( $400 \mathrm{U} / \mathrm{ml}$ in complete culture medium) overnight at room temperature on a rocker. The liberated cells were then centrifuged and washed once with PBS. The cells were centrifuged, and the cell pellets were resuspended in a $0.075 \mathrm{M} \mathrm{KCl}$ solution for $5 \mathrm{~min}$ and centrif uged again. The cell pellets were resuspended in Carnoy's fixative (methanol/glacial acetic acid ratio of 3). The fixative was changed once, and air-dried slides were prepared on wet microscope slides. The BAC DNA used as a probe was labeled with digoxigenin-deoxyUTP by nick translation. The labeled probe was combined with sheared mouse DNA (catalog number 18440-016; Invitrogen, Gaithersburg, MD) and hybridized to the fixed cells in a solution containing $50 \%$ formamide, $10 \%$ dextran sulfate, and $2 \times$ SSC $(0.03 \mathrm{~m}$ trisodium citrate, $\mathrm{pH} 7.0$, and $0.3 \mathrm{M}$ $\mathrm{NaCl}$ ). A specific probe signal was detected by incubating the hybridized slides in fluorescein-labeled antidigoxigenin antibodies followed by counterstaining with 4',6-diamidino-2-phenylindole. Our rapid interphase FISH genotyping results on $>163$ mice were consistent with previous metaphase FISH and PCR results (Zuo et al., 1999). To further determine the copy number of the BAC transgene, we performed quantitative FISH analysis on 40 interphase tail cells of one heterozygote (Poon et al., 1999). These experiments were performed using a CytoVision Image analysis system (Applied Image, Inc., Rochester, NY). All images were captured in one session to eliminate fluctuations in fluorescence attributable to fading of the dyes. The signal area was measured in pixels, and the signal intensity was measured in arbitrary units. The signal intensity was then multiplied by the signal area to yield a total fluorescence emission per signal. From a total of 40 interphase cells, the mean fluorescence emission for the transgene was divided by the mean fluorescence emission from the endogenous locus to estimate the copy number of the transgene relative to the endogenous locus. Because the endogenous signal represents a single copy of the BAC, the ratio of the endogenous signal and the transgenic signal would indicate the copy number of the BAC transgene. We obtained the ratio of $3.6 \pm 1.4$ (mean $\pm \mathrm{SD})(n=40)$. Thus, the copy number of the BAC transgene is $\sim 4$. Similar measurements on the BAC transgenic line mK9E4 showed that the copy number of the BAC transgene is $\sim 1$ (data not shown).

Western blot analysis. Mouse cochleas were homogenized in $0.5 \mathrm{ml}$ of radioimmunoprecipitation assay buffer $(0.15 \mathrm{M} \mathrm{NaCl}, 0.05 \mathrm{M}$ Tris, $0.1 \%$ NP-40, $0.05 \%$ deoxycholate, and $0.01 \%$ SDS). Equal volumes of each cochlea ( $40 \mu \mathrm{l} ; \sim 40 \mu \mathrm{g}$ of tissue) and $30 \mu \mathrm{g}$ of control tissues (i.e., brain, skeletal muscle, pituitary, and eye) were separated by SDS-PAGE and electroblotted to Immobilon P membranes (Millipore, Bedford, MA), and Western blotting was performed as described in the fast-blot protocol for Immobilon P membranes using either an antibody generated against $\alpha 9 \mathrm{nAChR}$ (MU43f) or an antimyosin VI antibody. The resulting autoradiographs were scanned, and band densities were determined using BioMax ID image analysis software (Eastman Kodak, Rochester, NY). To ensure that this semiquantitative analysis remained in the dynamic range of the measurement system, band densities were analyzed for a 10-fold dilution series performed on cochlear samples from both wild-type and transgenic ears.

Coimmunoprecipitation from mouse cochleas. Mouse cochleas were frozen and placed in PTN50 buffer (50 mM sodium phosphate, $\mathrm{pH} 7.4$, $1 \%$ Triton $\mathrm{X}-100$, and $50 \mathrm{~mm} \mathrm{NaCl}$ ) containing protease inhibitors and homogenized at high speed in a Brinkmann Polytron (Brinkmann Instruments, Westbury, NY) for $30 \mathrm{sec}$. The homogenate was freezethawed, and the lysate was centrifuged at $12,000 \times g$ in a microfuge for 5 min to pellet cellular debris. $\alpha 9 \mathrm{nAChRs}$ were immunoprecipitated from the lysate overnight at $4^{\circ} \mathrm{C}$ using the antibody MU43f ( $\alpha 9$ rabbit polyclonal); $\alpha 10 \mathrm{nAChRs}$ were immunoprecipitated from the lysate using the antibody MU81 ( $\alpha 10$ chicken polyclonal). The immune complexes were captured using immobilized protein A-Sepharose beads (Sigma, St. Louis, MO) or anti-chicken IgY-conjugated Sepharose beads (Promega, Madison, WI); the immunoprecipitated material was extracted by boiling in SDS Laemmli sample buffer. The samples were resolved by $4-15 \%$ SDS-PAGE and analyzed by Western blot analysis using antibody MU81 (anti- $\alpha 10$ antibody) or MU43 (anti- $\alpha 9$ antibody).

Auditory brainstem responses. Auditory brainstem responses (ABRs) were measured in each animal both before and after the acoustic overexposure. For the measurement, mice were anesthetized with xylazine $(20 \mathrm{mg} / \mathrm{kg}$, i.p.) and ketamine (100 mg/kg, i.p.). Needle electrodes were inserted at vertex and pinna, with a ground near the tail. ABR potentials were evoked with $5 \mathrm{msec}$ tone pips $\left(0.5 \mathrm{msec}\right.$ rise-fall with a $\cos ^{2}$ onset envelope, delivered at $35 / \mathrm{sec})$. The response was amplified $(10,000 \times)$, filtered $(0.1-3 \mathrm{kHz}$ ), and averaged with an analog-to-digital board in a LabVIEW-driven data-acquisition system (National Instruments, Austin, TX). The sound level was raised in $5 \mathrm{~dB}$ steps from $10 \mathrm{~dB}$ below threshold up to $80 \mathrm{~dB}$ sound pressure level (SPL). At each sound level, 1024 responses were averaged (with stimulus polarity alternated), using an "artifact reject," whereby response waveforms were discarded when the peak-to-peak amplitude was $>15 \mu \mathrm{V}$. On visual inspection of stacked waveforms, the threshold was defined as the lowest SPL level at which any wave could be detected, usually corresponding to the step just below that at which the peak-to-peak response amplitude rose significantly above the noise floor $(\sim 0.25 \mu \mathrm{V})$.

Cochlear immunostaining and quantification of immunopositive terminals. After intracardial perfusion with $10 \%$ formalin, cochleas were decalcified in EDTA and half-turns were dissected and immunostained as whole mounts. Tissue was incubated in primary antisera overnight [rabbit anti-vesicular acetylcholine transporter (VAT); Sigma], followed by a biotinylated secondary antibody, avidin-biotin-HRP complex (ABC kit; Vector Laboratories, Burlingame, $\mathrm{CA}$ ) and $\mathrm{DAB} / \mathrm{H}_{2} \mathrm{O}_{2}$; the tissue was then embedded in plastic and mounted on glass slides. For each cochlea, each dissected piece was measured by computerized planimetry and the cochlear location was converted to frequency (Ehret, 1983). To quantify immunopositive terminals, outlines were traced via a drawing tube using high-numerical-aperture objectives $(2000 \times$ total magnification). When tracing, the fine focus was continually adjusted to optimize the imaging of each terminal cluster. The traces were digitized, and the areas were computed with NIH Image software. In the OHC area, all immunopositive terminals were traced; the values from each row were averaged within bins corresponding to $100 \mu \mathrm{m}$ of cochlear length.

Distortion product otoacoustic emissions-based assay of OC-mediated suppression. Animals were anesthetized as for ABR testing, and posterior craniotomy and partial cerebellar aspiration were performed to expose the floor of the IVth ventricle. To stimulate the OC, shocks (monophasic pulses of $150 \mu \mathrm{sec}$ duration presented at 200 per sec) were applied through fine silver wires $(0.4 \mathrm{~mm}$ spacing $)$ placed along the midline, spanning the OC decussation. The shock threshold for facial twitches was determined, muscle paralysis was induced with $\alpha$-D-tubocurarine (1.25 $\mathrm{mg} / \mathrm{kg}$, i.p.), and the animal was connected to a respirator via a tracheal cannula. Shock levels were raised to $6 \mathrm{~dB}$ above the twitch threshold. The distortion product otoacoustic emission (DPOAE) at $2 f_{1}-f_{2}\left[f_{1}\right.$ and $f_{2}$ are the values of the two primary frequencies presented to the ear $\left(f_{2}>\right.$ $\left.f_{1}\right)$ ] was recorded with a custom acoustic assembly consisting of two 0.25 inch condenser microphones to generate primary tones $\left(f_{1}\right.$ and $f_{2}$ with $f_{2} / f_{1}=1.2$ and an $f_{2}$ level $10 \mathrm{~dB}$ less than the $f_{1}$ level) and a Knowles miniature microphone (EK3103; Knowles Electronics, Franklin Park, IL) to record sound pressure in the ear canal. Stimuli were generated digitally (AO-6; National Instruments, Austin, TX). The sound pressure in the ear canal was amplified and digitally sampled at $20 \mu \mathrm{sec}$ (A-2000; National Instruments). Fast Fourier transforms were computed and averaged over five consecutive waveform traces, and $2 f_{1}-f_{2}$ DPOAE amplitude and the surrounding noise floor were extracted, a procedure requir- 


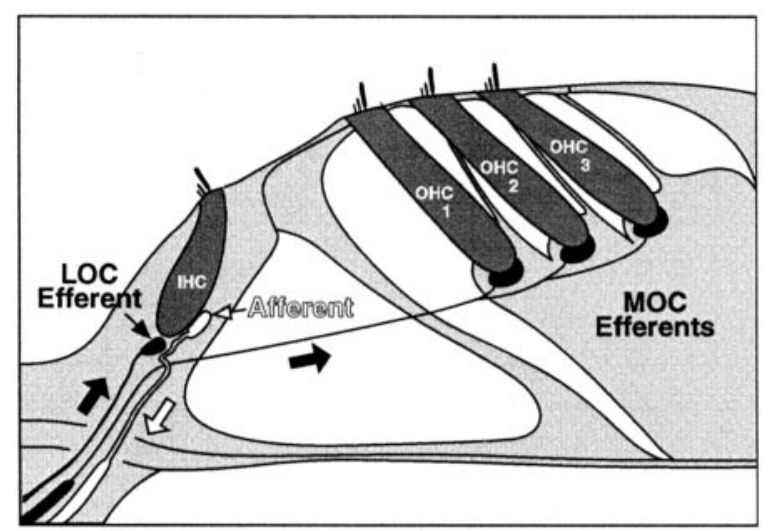

Figure 1. Cross section of the sensory epithelium of the inner ear (organ of Corti) showing one row of IHCs, three rows of OHCs, a single auditory nerve afferent contacting an IHC, a representative efferent fiber from the medial OC $(M O C)$ system, contacting all three rows of OHCs, and an efferent fiber from the lateral OC $(L O C)$ system contacting the peripheral terminal of an auditory nerve fiber. Arrows indicate the direction of information propagation along the neurons.

ing $\sim 6 \mathrm{sec}$ of data acquisition and processing time. DPOAE thresholds were defined by the visual inspection of amplitude versus level functions as the lowest $f_{2}$ level above which the DPOAE amplitude was always greater than the surrounding noise floor (which varied from -20 to $0 \mathrm{~dB}$ SPL, depending on the frequency; threshold amplitudes were typically $3-4 \mathrm{~dB}$ above the noise). During the OC suppression assay, the $f_{2}$ level was set to produce a DPOAE $\sim 10-15 \mathrm{~dB}$ greater than the noise floor. To measure OC effects, repeated measures of baseline DPOAE amplitude were first obtained $(n=12)$, followed by a series of 12 interleaved periods during which DPOAE amplitudes were measured with $(n=6)$ and without $(n=6)$ simultaneous shocks to the OC. The magnitude of the OC effect was defined as the mean decrease in DPOAE amplitude (in decibels) for all six trials that included shocks compared with the mean baseline amplitude.

Acoustic injury. Animals were exposed, awake, and unrestrained; they were kept within cages suspended inside a small reverberant soundexposure box (Liberman and Gao, 1995). The exposure stimulus was generated by a custom-made white-noise source, filtered with a 60 $\mathrm{dB} /$ octave slope, amplified (Crown power amp; Crown Audio, Elkhart, IN), and delivered (JBL compression driver; JBL Scientific, San Luis Obispo, CA) through an exponential horn fitted securely to a hole in the top of a reverberant box. Sound exposure levels were measured at four positions within each cage using a 0.25 inch Bruel and Kjaer (Copenhagen, Denmark) condenser microphone; the sound pressure was found to vary by $<0.5 \mathrm{~dB}$ across these measurement positions. The sound pressure was calibrated daily by positioning the microphone at the approximate position of the animal's head.

\section{RESULTS}

\section{Overexpression of $\alpha 9 \mathrm{nAChR}$ in transgenic animals}

In the present report, we studied a transgenic mouse line in which $\alpha 9$ is overexpressed via insertion of a modified BAC containing the mouse $\alpha 9 \mathrm{nAChR}$ gene and its promoter, along with a reporter gene, GFP (Fig. $2 A$ ). Mice were genotyped using both PCR and interphase FISH assays (Fig. 2B) (Zuo et al., 1999).

Transgenic animals showed normal growth and body weights, and their open-field behavior was indistinguishable from wildtype littermates. GFP expression in this transgenic line has been shown to recapitulate the endogenous expression of $\alpha 9 \mathrm{nAChR}$ during neonatal development, as determined by in situ hybridization (Zuo et al., 1999).

The pattern of transgene expression in the adult inner ear was investigated via endogenous fluorescence of the GFP reporter (Fig. 2C) and anti-GFP immunostaining (data not shown). Both methods suggested that $\alpha 9$ overexpression was driven only in
OHCs. There was no clear radial gradient among the three $\mathrm{OHC}$ rows; however, GFP signals decreased in intensity from apex to base.

Western blot analysis of cochlear homogenates (Fig. 2D-F) suggested that levels of $\alpha 9$ protein, normalized to hair-cell number, were $\sim 1.6$ times higher in transgenic mice than in wild-type littermates: expressed in arbitrary units of optical density, values of $1.33 \pm 0.07$ in wild types $(n=13)$ versus $1.98 \pm 0.21$ for heterozygotes $(n=6)$ versus $2.12 \pm 0.27$ for homozygotes $(n=6)$ were obtained (Fig. 2D). Differences between wild types and either transgenic genotype were statistically significant $(p<$ 0.001; Student's $t$ test); however, the small mean difference between the homozygous and heterozygous animals was not.

Coimmunoprecipitation studies (Fig. 3) suggest that overexpression of $\alpha 9$ protein led to the formation of more $\alpha 9 / \alpha 10$ complexes in cochlear tissues. When cochlear lysates were precipitated with a rabbit anti- $\alpha 9$ antibody and then probed with chick anti- $\alpha 10$ (Fig. $3 C$ ), the band density indicating $\alpha 10$ levels on the resultant gels was higher in transgenic cochleas than in wild types. In the example shown, the densities of the wild-type, heterozygote, and homozygote transgenic mice were 38.9, 61.9, and 76.8 arbitrary units, respectively. Similarly, when lysates were precipitated with anti- $\alpha 10$ and probed with anti- $\alpha 9$ (Fig. $3 B$ ), the band densities were higher in transgenic mice than in wild types. Similar results were obtained with two independent runs from different sets of cochleas for each of the two complementary coimmunoprecipitation tests. Control lanes show a lack of staining in antibody-only controls; the two tests were run identically, except that the controls were run without the cochlear lysates, to demonstrate the lack of cross-reactivity between the two primary antibodies themselves.

\section{Cochlear function before acoustic injury}

Assessment of baseline cochlear function, conducted in doubleblind manner, revealed no significant differences between transgenic mice and wild-type littermates. Cochlear function was assessed by measurement of both ABRs and DPOAEs. The ABR measurement represents synchronous neural activity generated at several levels of the ascending auditory pathways, including the auditory nerve. The DPOAEs are sounds created within the cochlea, amplified by the action of OHCs and propagated through the middle ear back to the ear canal, where they can be measured with a microphone (Kemp, 1986). Although the ear creates DPOAEs at a number of frequencies, the largest is that at the frequency $2 f_{1}-f_{2}$.

For ABRs and DPOAEs, data were gathered in such a way as to allow both a measure of the threshold of response (Fig. 4A,B) (i.e., the lowest stimulus level required to produce a criterion response chosen to be just above the measurement noise floor) and the growth of response magnitude with sound pressure level (Fig. 4C,D). For growth functions, data are shown at only one test frequency; however, the results were similar for all test frequencies measured (all frequencies tested can be read from Fig. 4A, $C$ ). Thus, the normality of all of these response measures in the transgenic animals indicates that cochlear mechanics, transduction, and synaptic transmission at low and moderate sound levels are not significantly altered by the $\alpha 9$ overexpression.

\section{OC morphology and function before acoustic injury}

A second set of pre-exposure baseline experiments demonstrated that classic efferent suppressive effects were significantly enhanced in the transgenic animals (Fig. 5). To evaluate the effects 


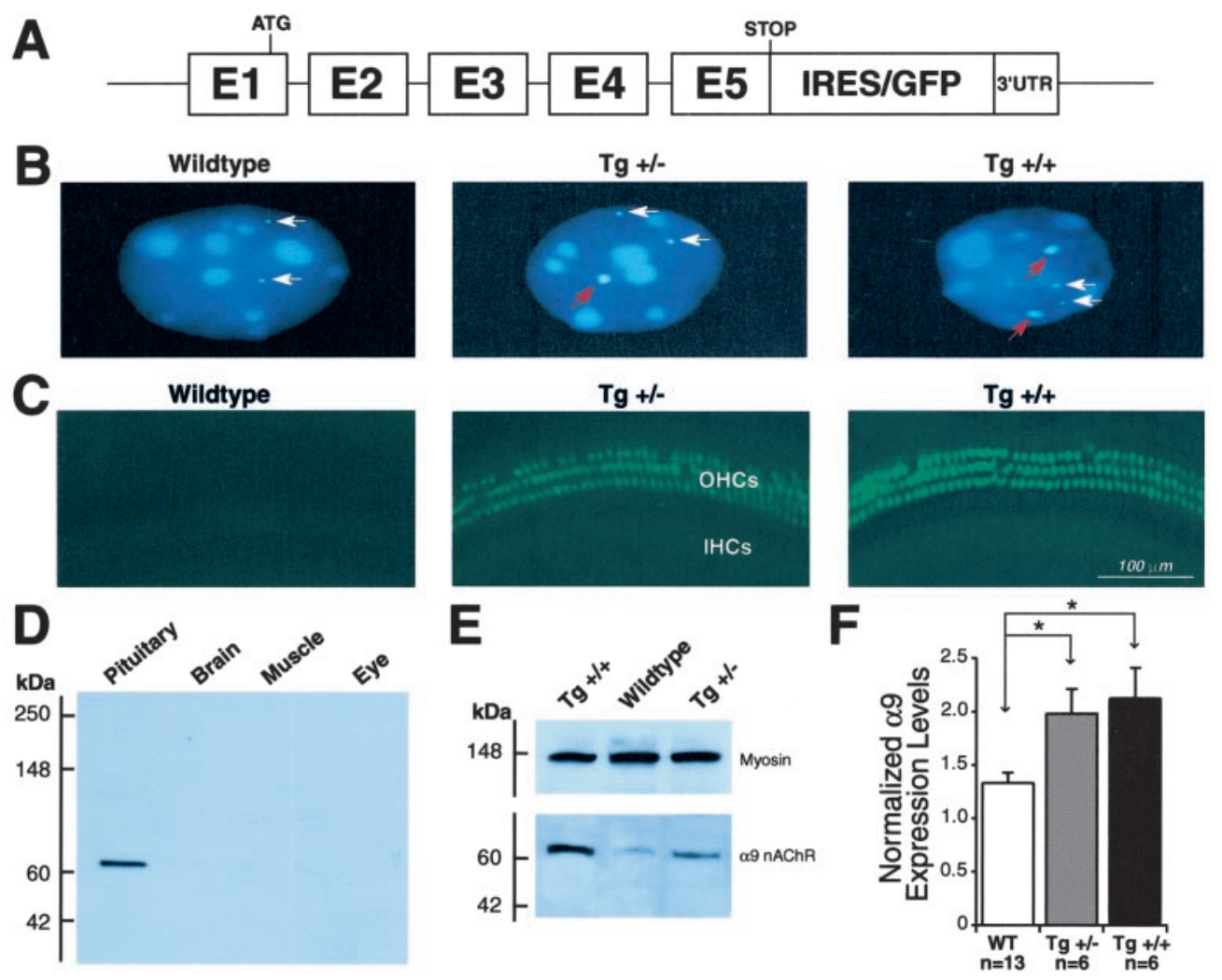

Figure 2. Construction and characterization of transgenic mice overexpressing $\alpha 9 \mathrm{nAChR}$. $A$, The schematic illustrates the site-directed mutagenesis of a BAC containing the mouse $\alpha 9 \mathrm{nAChR}$ gene. IRES, Internal ribosome entry site; UTR, untranslated region. $B$, FISH genotyping using mouse $13 \mathrm{~K} 9$ BAC DNA and interphase chromosomes of a cell from the tail of a 1-week-old wild-type, adult heterozygote $(\mathrm{Tg}+/-)$, or adult homozygote (Tg $+/+)$ mouse. The endogenous locus is indicated by the white arrows, and the transgenic locus is indicated by the red arrows. $C$, Fluorescence microscopy shows that expression of GFP reporter in transgenic animals is restricted to cochlear OHCs, with a stronger signal in homozygotes $(\mathrm{Tg}+/+)$ than in heterozygotes $(\mathrm{Tg}+/-)$. The images represent identical exposures of surface preparations of the organ of Corti from the apical half of the cochlea in adult mice from each of three genotypes. $D, E$, Western blots show $\alpha 9 \mathrm{nAChR}$ protein expression in transgenic mice restricted to the pituitary and cochlea; in the cochlea, the expression is stronger in transgenic animals than in wild-type littermates. $F$, Quantitative analysis of Western blots. Expression levels were quantified by measuring band density ( $60 \mathrm{kDa}$ band) and normalizing to myosin VI (148 $\mathrm{kDa}$ band). Error bars indicate means \pm SEM; $n$, number of animals in each sample; asterisks indicate statistical significance ( $p<0.001$; Student's $t$ test).

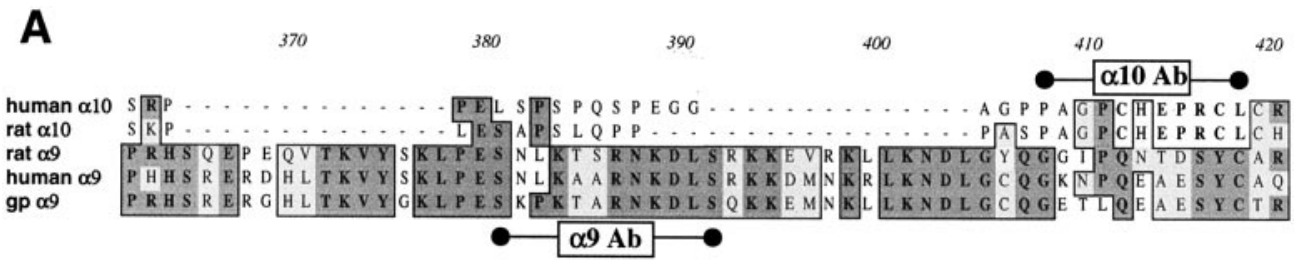

B ipt $\alpha 10 /$ detect $\alpha 9$

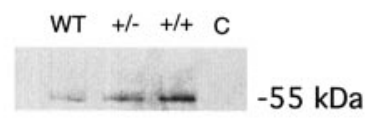

C ipt $\alpha 9 /$ detect $\alpha 10$

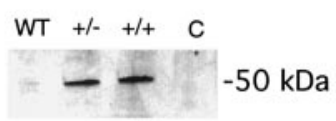

Figure 3. Coimmunoprecipitation tests show that there is an increase in $\alpha 9 / \alpha 10$ protein complexes in the transgenic cochleas. $A$, Fragments of the peptide alignments for $\alpha 9$ and $\alpha 10$ proteins from humans, rats, and guinea pigs illustrate the peptide regions used to create the anti- $\alpha 9$ and anti- $\alpha 10$ antibodies. Dark gray regions denote absolute identity with $\alpha 9$ from guinea pigs; light gray denotes conserved protein identity. $g p$, Guinea pig. $B$, $C$, Western blots for two complementary coimmunoprecipitation tests: precipitate with $\alpha 10$ and probe with $\alpha 9(A)$ and precipitate with $\alpha 9$ and probe with $\alpha 10$. Each data set is derived from cochlear lysates pooled from eight cochleas from four animals of each genotype. $W T$, Wild type; $+/+$, homozygotes; $+/-$, heterozygotes; $C$, controls; ipt, immunoprecipitation.

of $\alpha 9$ overexpression on these efferent effects, DPOAEs were measured while electrically stimulating the efferents at the floor of the IVth ventricle, where they run close to the surface of the brainstem (Warr et al., 1986). Given the close relationship between $\mathrm{OHC}$ function and distortion product amplitude, the de- gree of DPOAE suppression can be a sensitive measure of these cholinergic actions on OHCs. As illustrated in Figure $5 B$, our assay of classic efferent cochlear suppression involved repeated measures of DPOAE magnitude without (Fig. 5, open triangles) versus with (Fig. 5, closed triangles) simultaneous electrical acti- 
Figure 4. Characterization of baseline cochlear function in transgenic mice versus littermate wild types before acoustic overexposure. Group means \pm SEM are plotted; the numbers of animals in each group are indicated in the key. $A, C$, Pre-exposure thresholds for ABRs and ABR amplitude-versus-level functions are unaffected by the transgene. ABR thresholds are determined by visual inspection of waveforms obtained at $5 \mathrm{~dB}$ increments in SPL. ABR amplitude-versus-level functions are shown only for the test frequency at $22.62 \mathrm{kHz}$. B, D, DPOAE thresholds and amplitude-versus-level functions are also unaffected by the transgene. DPOAE thresholds are determined by visual inspection of waveforms obtained at $5 \mathrm{~dB}$ increments in SPL. Amplitude-versus-level functions are shown only for $f_{2}=14.14 \mathrm{kHz}$. $W T$, Wild type.

Figure 5. Efferent-mediated suppression of cochlear DPOAEs is enhanced in transgenic animals. $A$, Data show mean suppression \pm SEM of the DPOAE at $2 f_{1}-f_{2}$ attributable to efferent electrical stimulation. The numbers of animals tested in each group are indicated in the key. Techniques for deriving efferent effect magnitude are illustrated in $B$. Dashed line indicates no efferent effect. $B$, Measurement of efferent effect magnitude in one animal at $f_{2}=22.62 \mathrm{kHz}$. For all measurements, the $f_{2}$ level was set to produce a DPOAE $\sim 10-15 \mathrm{~dB}$ above the noise floor. Repeated measurements of baseline DPOAE amplitude were first obtained $(n=12)$, followed by a series of 12
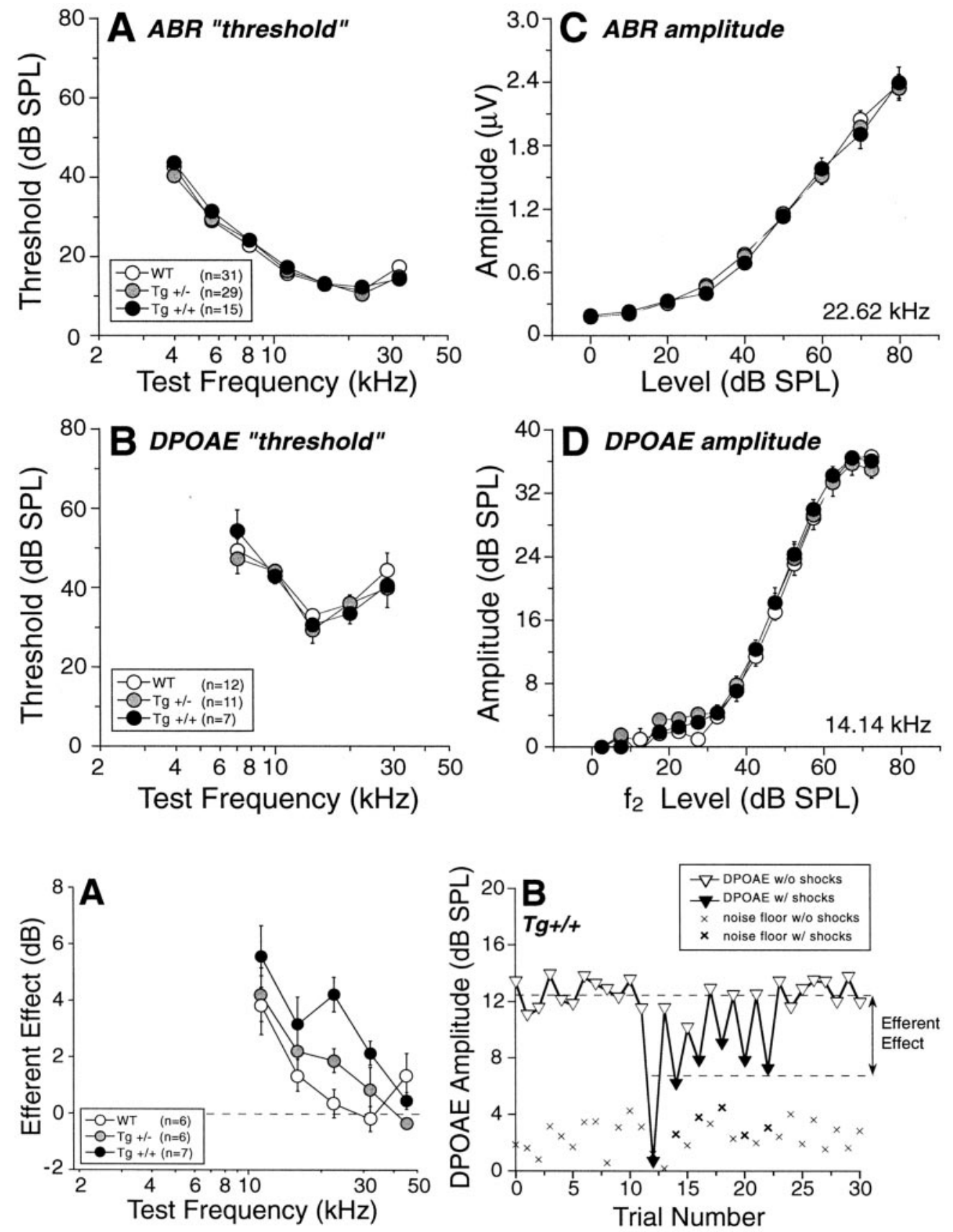

interleaved periods in which DPOAE amplitudes were measured with $(n=6)$ and without $(n=6)$ simultaneous shocks to the OC. The black arrow indicates the magnitude of the efferent effect, defined as the mean decrease in DPOAE amplitude (in decibels) for all six with-shock trials (bottom dashed line) compared with the mean baseline amplitude (top dashed line). The noise floor indicates the average spectral level for the six frequency points surrounding $2 f_{1}-f_{2}$. WT, Wild type.

vation of the efferent bundle with 200 shock trains per second. The efferent effect was then defined as the difference (in decibels) between the average DPOAE amplitude for the 12 trials before the first shock trains and the average DPOAE amplitude for the six trials during shocks to the efferent bundle. Note that for the individual data run shown in Figure $5 B$, the efferent effect magnitude tends to decrease with repeated stimulation. Similar adaptation of OC-mediated cochlear suppression has been reported previously (Wiederhold and Kiang, 1969).

The data in Figure $5 A$ summarize the mean effects seen in a number of wild-type versus transgenic animals. These mean data show that $\alpha 9$ overexpression increased the magnitude of efferentmediated cochlear suppression for test frequencies between 16 and $32 \mathrm{kHz}$ (two-way ANOVA; $F_{(1,13)}=8.961 ; p=0.01$ ), with smaller effects seen above and below that region.

To determine whether these enhanced peripheral effects in the transgenic line arise from fundamental alterations in efferent innervation patterns, the size and number of cholinergic OC terminals on OHCs were quantified in one homozygous transgenic animal (Fig. 6). To visualize these terminals, cochleas were immunostained for VAT, and the silhouette areas of immunopositive terminals were measured throughout the cochlear spiral. As 


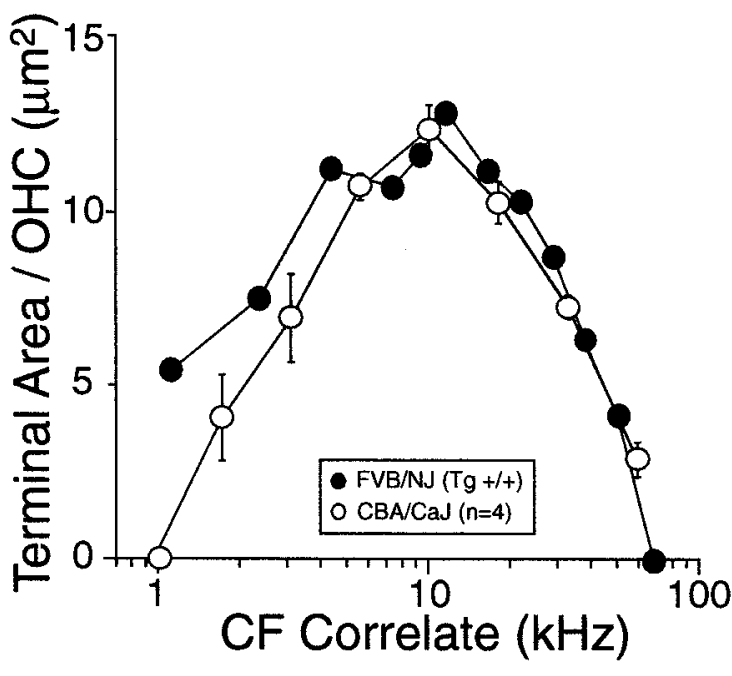

Figure 6. Density of cholinergic OC terminals on OHCs as a function of cochlear location for transgenic versus normal mice. Data show the total silhouette area of immunostained terminals (anti-VAT) from all three rows. Transgenic data are from a single homozygous animal. Control data were obtained from four CBA/CaJ mice. Error bars indicate SEM. Innervation densities are expressed as total silhouette area per OHC. $C F$, Cochlear frequency.

shown in Figure 6, this estimate of total terminal volume for the cholinergic innervation of OHCs normally peaks in the middle of the cochlear spiral, at cochlear regions tuned near $12 \mathrm{kHz}$. Results from the transgenic cochlea were not significantly different.

\section{Protection from acoustic injury}

To evaluate the effects of $\alpha 9$ overexpression on the vulnerability to both reversible and irreversible forms of acoustic injury, groups of transgenic and wild-type animals were exposed for $2 \mathrm{hr}$ to a noise band $(8-16 \mathrm{kHz})$ at either 92 or $110 \mathrm{~dB}$ SPL and allowed to recover for $12 \mathrm{hr}$ or $1 \mathrm{wk}$ before assessing the degree of temporary (Fig. 7) or permanent noise-induced hearing loss (Fig. 8), respectively, via measurement of thresholds for auditory evoked potentials. All exposures and subsequent physiological analyses were performed by the Boston-based team, who were kept unaware of the genotyping data.

When measured $12 \mathrm{hr}$ after exposure at $92 \mathrm{~dB}$, temporary threshold shifts (TTSs) were maximal near $16 \mathrm{kHz}$ for all genotypes (Fig. 7A); 1 wk later, mean thresholds had returned to normal (data not shown), demonstrating the reversibility of the damage from the $92 \mathrm{~dB}$ exposure. The mean TTS was significantly smaller in transgenic than in wild-type animals over the entire range of tested frequencies (two-way ANOVA; $F_{(1,12)}=$ $10.03 ; p=0.008)$. Pairwise comparisons between either heterozygote or homozygote groups and wild types also showed statistically significant differences (two-way ANOVA; $F_{(1,10)}=7.293$, $p=0.022$, and $F_{(1,6)}=21.251, p=0.004$, respectively). Although the trend of the means suggests that homozygotes were slightly more resistant than heterozygotes, the differences were not significant. When viewed as amplitude-versus-level functions, ABR data show consistently higher suprathreshold response amplitudes for transgenic versus wild-type animals. Three octave-spaced test frequencies are shown (Fig. 7B,C); however, the results were similar for the other test frequencies measured $(8,16$, and 32 $\mathrm{kHz}$ ).

The $110 \mathrm{~dB}$ exposure produced significantly more acute threshold shift, and it resolved, among wild-type controls, to a perma- nent threshold shift (PTS) of 15-25 dB across all frequencies tested, when measured 1 week after exposure (Fig. $8 A$ ). Among the animals exposed at $110 \mathrm{~dB}$, there was also a clear reduction in PTS vulnerability among the transgenic animals (two-way ANOVA; $\left.F_{(1,12)}=5.968 ; p=0.032\right)$. By chance, the littermates used in this arm of the study contained no animals homozygous for the transgene. Measurements of the growth of response magnitude with SPL also revealed consistent and dramatic differences between genotypes, with higher response amplitudes at all levels for transgenic mice. Three octave-spaced test frequencies are illustrated in Figure $8 B, C$; similar results were seen at three other test frequencies $(8,16$, and $32 \mathrm{kHz})$

Two previous experimental series also showed significantly enhanced resistance to acoustic injury in transgenic animals (data not shown). One series (with five wild-type, three $\mathrm{Tg}+/-$, and six $\mathrm{Tg}+/+$ animals) produced a slightly more severe TTS than that shown in Figure $7 A$ (noise band at 94 instead of $92 \mathrm{~dB}$ ). A second series (with 12 wild-type, $11 \mathrm{Tg}+/-$, and $7 \mathrm{Tg}+/+$ animals) produced a smaller PTS than that shown in Figure $8 A$ (noise band at 104 instead of $110 \mathrm{~dB}$ ). In the second series, PTS differences between wild type and each transgenic genotype were statistically significant in the range $4-22.62 \mathrm{kHz}$ by ANOVA $\left(F_{(1,21)}=5.587, p=0.028\right.$ for wild type vs $\mathrm{Tg}+/-$ and $F_{(1,17)}=$ $4.795, p=0.043$ for wild type vs $\mathrm{Tg}+/+)$, whereas differences between heterozygous and homozygous animals were not $\left(F_{(1,16)}\right.$ $=0.120 ; p>0.05)$.

A final series of control experiments was performed to exclude a possible contribution to the observed resistance of other factors, such as the expression of GFP or other genes in the BAC construct. To this end, a control BAC construct was designed differing only by deletion of exon 4 of the $\alpha 9 \mathrm{nAChR}$ gene (Fig. $2 A)$. This new transgenic line expressed GFP without overexpressing the $\alpha 9$ protein (as quantified by Western blot), and after exposure to the $110 \mathrm{~dB}$ noise band showed a PTS pattern that was statistically indistinguishable from that seen in wild-type animals (data not shown).

\section{DISCUSSION}

These results represent a key step in dissecting the contributions of different transmitter/receptor complexes to the observed effects of the structurally and cytochemically diverse OC efferent system. Data from these mutant mice provide direct evidence that efferent-mediated protection from acoustic injury is mediated via the $\alpha 9 \mathrm{nAChR}$ complexes on OHCs. Furthermore, the fact that the magnitude of the heightened resistance associated with $\alpha 9$ overexpression is comparable with the magnitude of the heightened PTS vulnerability seen after surgical de-efferentation $(\mathrm{Ku}-$ jawa and Liberman, 1997) and with the increased TTS resistance associated with massive electrical stimulation of the efferent bundle (Rajan, 1988) is consistent with the idea that $\alpha 9$ participates directly in all of the efferent-mediated protective effects observed to date. For example, in the pioneering study by Rajan (1988) of electrically evoked OC activity and TTS in guinea pigs, the largest protection reduced peak TTS by only $16 \mathrm{~dB}$ (from $\sim 22 \mathrm{~dB}$ in control ears to $\sim 6 \mathrm{~dB}$ in ears with $400 / \mathrm{sec}$ shocks to the OC bundle during acoustic overexposure). In comparison, the transgenic overexpression of $\alpha 9$ in the present study reduced TTS by as much as $22 \mathrm{~dB}$ (compare $22 \mathrm{kHz}$ values for wild-type vs transgenic animals in Fig. $7 A$ ).

The additional observation that $\alpha 9$ overexpression was restricted to the OHCs also strongly implicates these cells, and thus the medial OC system, in the phenomenon of efferent-mediated 
Figure 7. $\alpha 9 \mathrm{nAChR}$ overexpression reduces temporary noise-induced damage. $A$, Protective effects of the transgene for an exposure designed to cause TTS: $2 \mathrm{hr}$ of exposure to an $8-16 \mathrm{kHz}$ noise band at 92 dB SPL. When measured $12 \mathrm{hr}$ after exposure, the mean TTS was significantly larger in wild types than in transgenics. $W T$, Wild type. $B, C, A B R$ amplitude-versus-level functions are shown for two test frequencies. Data are expressed as group means \pm SEM. Gray band indicates passband of the noise used for acoustic overstimulation. Dashed line indicates no threshold shift.

Figure 8. $\alpha 9$ nAChR overexpression reduces permanent noise-induced damage. $A$, Protective effects of the transgene for an exposure designed to cause PTS: $2 \mathrm{hr}$ of exposure to an $8-16 \mathrm{kHz}$ noise band at 110 dB SPL. When measured 1 wk after exposure, the mean PTS was significantly larger in wild-type than in transgenic animals. Data are expressed as group means \pm SEM. $W T$, Wild type. $B, C$, ABR amplitudeversus-level functions are shown for two test frequencies. Data are expressed as group means \pm SEM. Gray band indicates passband of the noise used for acoustic overstimulation. Dashed line indicates no threshold shift.
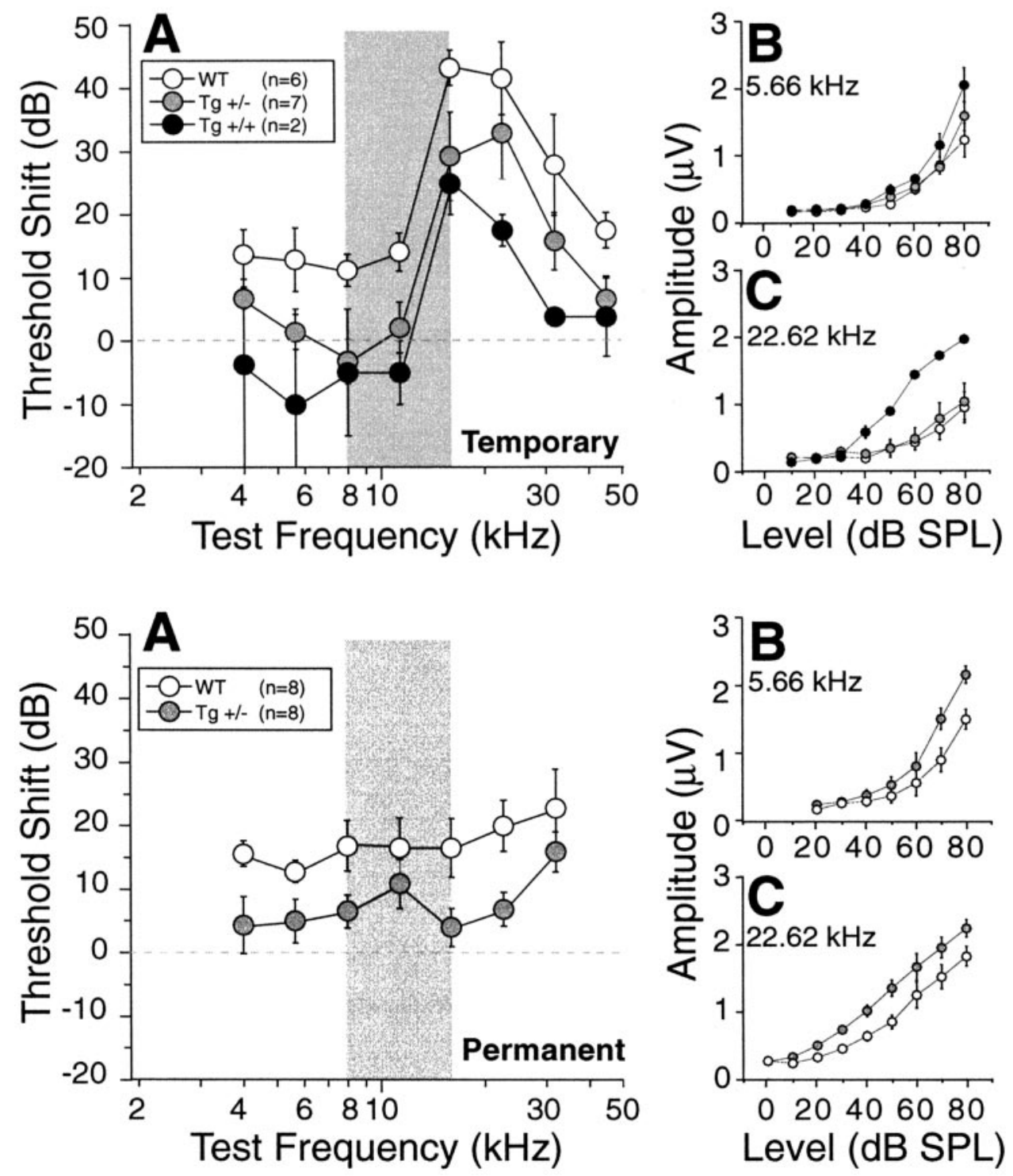

protection. Although in situ hybridization studies have shown a clear $\alpha 9$ signal in the IHCs of adult rats (Simmons and Morley, 1998; Luo et al., 1998), the native expression pattern in adult mice has never been investigated; the lack of $\alpha 9$ expression in our transgenic mice is consistent with the transition of efferent innervation from IHCs to OHCs during postnatal development. Thus, the present data show that there is no reason to invoke any noncholinergic effects of the medial OC system on OHCs, nor any action of the lateral OC system in the IHC area to explain OC-mediated protection from acoustic injury.

Work on the $\alpha 9$ knock-out mouse suggests that the $\alpha 9 \mathrm{nAChR}$ is also essential for the generation of classic efferent-mediated cochlear suppression of responses to low and moderate sound levels (Vetter et al., 1999). The present results are consistent in that overexpression of $\alpha 9$ led to enhancements of this type of efferent-mediated suppression (Fig. 5). Thus, both of the bestcharacterized efferent effects appear interrelated via a common dependence on the $\alpha 9$ subunit. The observed frequency dependence of OC suppressive effects (Fig. $5 A$ ) is consistent with the longitudinal distribution of $\mathrm{OC}$ cholinergic terminals in the mouse (Fig. 6): both appear to peak at approximately the $12 \mathrm{kHz}$ region. Our measures of OC suppression are limited to frequencies of $>11.3 \mathrm{kHz}$, because DPOAE amplitudes in mice are very small for $f_{2}$ at $\leq 8 \mathrm{kHz}$ (S. F. Maison and M. C. Liberman, unpublished observations). Thus, the correlation between the frequency dependence of the peripheral effects of $\mathrm{OC}$ and the cochlear distribution of OC terminals is not as compelling as in cats or guinea pigs (Liberman et al., 1990). The apparent frequency dependence of the enhancements of OC suppression in the transgenic mice (i.e., largest at $22.6 \mathrm{kHz}$ ) (Fig. 5A) must be viewed cautiously. In particular, the effects of $\alpha 9$ overexpression may be underestimated at $11.3 \mathrm{kHz}$, at which OC effects peak, because of saturation in the assay. To maximize OC effects, we minimized the sound pressure of the primaries, because OC effects are maximal near threshold (Wiederhold, 1970). Thus, the primary levels were set to produce a DPOAE only $10 \mathrm{~dB}$ above the noise floor, and, when the OC effects were large, DPOAEs were often driven into the noise (e.g., first point with OC shocks) (Fig. 5B), thus saturating the assay and reducing measured enhancements.

Enhanced OC effects in the transgenic animals are not attributable to fundamental changes in the efferent innervation pattern 
associated with $\alpha 9$ overexpression (Fig. 6). Thus, they are presumed to arise from changes in the number, nature, or distribution of $\alpha 9$ receptor complexes in the postsynaptic membrane. Evidence suggests that $\alpha 9$ in OHCs in vivo is complexed with $\alpha 10$ nAChR subunits: in vitro coexpression of $\alpha 10$ with $\alpha 9$ produces ligand-gated currents and desensitization behavior more like native receptors; $\alpha 9$ homomers show smaller currents but less desensitization (Elgoyhen et al., 2001). Our coimmunoprecipitation data (Fig. 3) show that the $\alpha 9$ upregulation driven by the transgene is coupled with an increased formation of $\alpha 9 / \alpha 10$ complexes in the cochlea. Thus, the enhancement of classic OC suppression, observed in transgenic animals (Fig. 5), is well explained by an increased level of functional $\alpha 9 / \alpha 10$ complexes. Whereas $\alpha 9$ mRNA in our transgenic cochleas was increased by more than fivefold with regard to wild-type animals (Zuo et al., 1999), $\alpha 9$ protein was increased only 1.5-1.7 times (Fig. 2F). Although Western blot estimates of expression changes are semiquantitative at best, increases in protein expression comparable with the mRNA enhancement may be limited by space constraints at the postsynaptic membrane. Transgenic overexpression studies of rhodopsin in retinal photoreceptor cells have also noted modest (i.e., 20-25\%) changes in protein levels despite clear-cut changes in the phenotype (Tan et al., 2001).

The idea that $\alpha 9$ overexpression could have dramatic effects on the response of the ear to high-level sounds (Figs. 7, 8), with no changes in threshold sensitivity or responses to moderate-level stimuli (Fig. 4), fits well with the known characteristics of this sound-evoked feedback pathway. The efferent neurons that project to these cholinergic synapses on OHCs have low levels of spontaneous activity and begin to respond to sound at levels 20-30 dB above afferent fiber thresholds (Liberman, 1988). As the sound level increases, efferent discharge rates increase monotonically; thus the magnitude of their peripheral effects should also increase with increasing sound level.

There are two fundamentally different ways in which $\alpha 9$ activation could reduce acoustic injury: (1) directly, via protective effects on the OHCs themselves, because these cells are both necessary for the exquisite threshold sensitivity and are among the elements most vulnerable to acoustic overexposure, or (2) indirectly, by reducing the $\mathrm{OHC}$ contribution to amplification of mechanical vibrations of the cochlear sensory epithelium.

The case for mechanical effects (hypothesis 2) lacks key empirical data. Although OC activation can reduce cochlear vibrations to low-level sounds (Murugasu and Russell, 1996), evidence concerning the magnitude of these efferent effects for high levels of acoustic stimulation is sparse and contradictory (Guinan and Stankovic, 1996). Clarifying these mechanical effects is key to understanding the mechanisms. In all previously studied mammalian models of acoustic injury (including FVB/ $\mathrm{N}$ mice), the magnitude of noise-induced PTS grows by 5-10 dB for every $1 \mathrm{~dB}$ increase in exposure level once a damaging exposure level is reached (Yoshida et al., 2000; see below). Thus, efferent protective effects as large as $30 \mathrm{~dB}$ can arise from a suppression of cochlear vibration equivalent to only a $3 \mathrm{~dB}$ decrease in the input stimulus level.

The alternative hypothesis (i.e., that $\alpha 9$ activation leads directly to hair cell protection) is interesting in light of the hypothesized role of the $\alpha 7$ subunit in mediating a variety of neuroprotective effects in vitro (Cordero-Erausquin et al., 2000). Previous studies of OC effects in vivo have suggested that $\alpha 9$ activation leads to both a rapid $\left(\tau=100 \mathrm{msec}\right.$ ) calcium-activated $\mathrm{K}^{+}$efflux as well as a slow $(\tau=10 \mathrm{sec})$ wave of calcium-induced calcium release in hair cells
(Sridhar et al., 1997). Additional circumstantial evidence has linked the magnitude of this slow effect with the magnitude of OCmediated protection (Reiter and Liberman, 1995).

According to the present conclusions, the $\alpha 9$ knock-out mouse should be exceptionally vulnerable to acoustic injury. A previous attempt to address this question (Yoshida et al., 1999) failed to show significant effects of the knock-out on acoustic vulnerability. However, the study was inconclusive about the role of $\alpha 9$, because it was also shown that the knock-out background strain (129/ $\mathrm{SvEv}$ ) is uniquely resistant to acoustic injury (Yoshida et al., 2000). In 129/SvEv mice, the growth rate of PTS with exposure level is only $1 \mathrm{~dB} / \mathrm{dB}$, rather than the $5-10 \mathrm{~dB} / \mathrm{dB}$ discussed above. Thus, if efferent-mediated protective effects arise via soundevoked feedback inhibition of cochlear mechanical vibration equivalent to only $1-2 \mathrm{~dB}$ of stimulus reduction, the effects on PTS will not appear significant in the uniquely resistant $129 / \mathrm{SvEv}$ ear, in which that small effective attenuation is not amplified into a PTS reduction 5-10 times larger.

\section{Conclusions}

Our investigations provide direct evidence that efferent-mediated protection from acoustic injury is mediated via the $\alpha 9 \mathrm{nAChR}$ complexes on OHCs. The present results focus future investigation of efferent-mediated protection on clarification of the downstream effects of ligand binding to the $\alpha 9$ receptors. In a broader context, the results also constitute the first direct demonstration of in vivo modulation of a protective effect of nAChRs by transgenic modulation of the expression level of a particular receptor subunit. The fact that $\alpha 9$ receptor expression is limited to very few sites in the nervous system and the fact that the transgenic overexpression in the ear can be clearly limited to one cell class provide a unique opportunity to isolate and characterize the role of one member of the $\mathrm{nAChR}$ receptor subunit family in the generation of a protective effect in vivo.

\section{REFERENCES}

Cordero-Erausquin M, Marubio LM, Klink R, Changeux J-P (2000) Nicotinic receptor function: new perspectives from knockout mice. Trends Pharmacol Sci 21:211-217.

Dallos P (1992) The active cochlea. J Neurosci 12:4575-4585.

Dolan DF, Nuttall AL (1988) Masked cochlear whole-nerve response intensity functions altered by electrical stimulation of the crossed olivocochlear bundle. J Acoust Soc Am 83:1081-1086.

Ehret G (1983) Peripheral anatomy and physiology II. In: The auditory psychobiology of the mouse (Willott JF, ed), pp 169-200. Springfield, IL: Thomas.

Elgoyhen AB, Johnson DS, Boulter J, Vetter DE, Heinemann S (1994) $\alpha 9$ : an acetylcholine receptor with novel pharmacological properties expressed in rat cochlear hair cells. Cell 79:705-715.

Elgoyhen AB, Vetter DE, Katz E, Rothlin CV, Heinemann SF, Boulter J (2001) $\alpha 10$ : a determinant of nicotinic cholinergic receptor function in mammalian vestibular and cochlear mechanosensory hair cells. Proc Natl Acad Sci USA 98:3501-3506.

Eybalin M (1993) Neurotransmitters and neuromodulators of the mammalian cochlea. Physiol Rev 73:309-373.

Fuchs PA, Murrow BW (1992) Cholinergic inhibition of short (outer) hair cells of the chick's cochlea. J Neurosci 12:800-809.

Guinan JJ, Stankovic KM (1996) Medial efferent inhibition produces the largest equivalent attenuations at moderate to high sound levels in cat auditory-nerve fibers. J Acoust Soc Am 100:1680-1690.

Housley GD, Ashmore JF (1991) Direct measurement of the action of acetylcholine of isolated outer hair cells of the guinea pig cochlea. Proc R Soc Lond B Biol Sci 244:161-167.

Jones S, Sudweeks S, Yakel JL (1999) Nicotinic receptors in the brain: correlating physiology with function. Trends Neurosci 22:555-561.

Kemp DT (1986) Otoacoustic emissions, travelling waves, and cochlear mechanisms. Hear Res 22:95-104.

Kujawa SG, Liberman MC (1997). Conditioning-related protection from acoustic injury: effects of chronic de-efferentation and sham surgery. J Neurophysiol 78:3095-3106.

Liberman MC (1988) Physiology of cochlear efferent and afferent neurons: direct comparisons in the same animal. Hear Res 34:179-192. 
Liberman MC, Gao WY (1995) Chronic cochlear de-efferentation and susceptibility to permanent acoustic injury. Hear Res 90:158-168.

Liberman MC, Dodds LW, Pierce S (1990) Afferent and efferent innervation of the cat cochlea: quantitative analysis using light and electron microscopy. J Comp Neurol 301:443-460.

Luo L, Bennett T, Jung HH, Ryan AF (1998) Developmental expression of $\alpha 9$ acetylcholine receptor mRNA in the rat cochlea and vestibular inner ear. J Comp Neurol 393:320-331.

Murugasu E, Russell IJ (1996) The effect of efferent stimulation on basilar membrane displacement in the basal turn of the guinea pig cochlea. J Neurosci 16:325-332.

Poon SS, Martens UM, Ward RK, Lansdorp PM (1999) Telomere length measurements using digital fluorescence microscopy. Cytometry $36: 267-278$

Rajan R (1988) Effect of electrical stimulation of the crossed olivocochlear bundle on temporary threshold shifts in auditory sensitivity. I. Dependence on electrical stimulation parameters. J Neurophysiol 60: 549-568.

Reiter ER, Liberman MC (1995) Efferent-mediated protection from acoustic overexposure: relation to slow effects of olivocochlear stimulation J Neurophysiol 73:506-514.

Simmons DD, Morley BJ (1998) Differential expression of the $\alpha 9$ nicotinic acetylcholine receptor subunit in neonatal and adult cochlear hair cells. Brain Res Mol Brain Res 56:287-292.

Sridhar TS, Brown MC, Sewell WF (1997) Unique postsynaptic signaling at the hair cell efferent synapse permits calcium to evoke changes on two time scales. J Neurosci 17:428-437.
Tan E, Wang Q, Quiambao AB, Xu X, Qtaishat NM, Peachey NS, Lem J, Fliesler SJ, Pepperberg DR, Naash MI, Al-Ubaid MR (2001) The relationship between opsin overexpression and photoreceptor degeneration. Invest Ophthalmol Vis Sci 42:589-600.

Vetter DE, Liberman MC, Mann J, Barhanin J, Boulter J, Brown MC, Saffiote-Kolman J, Heinemann SF, Elgoyhen AB (1999) Role of $\alpha 9$ nicotinic $\mathrm{ACh}$ receptor subunits in the development and function of cochlear efferent innervation. Neuron 23:93-103.

Warr WB, Guinan JJ, White JS (1986) Neurobiology of hearing: the cochlea. New York: Raven.

Wiederhold ML (1970) Variations in the effects of electric stimulation of the crossed olivocochlear bundle on cat single auditory nerve fiber responses to tone bursts. J Acoust Soc Am 48:966-977.

Wiederhold ML, Kiang NYS (1969) Effects of electric stimulation of the crossed olivocochlear bundle on single auditory-nerve fibers in the cat. J Acoust Soc Am 48:950-965.

Yoshida N, Vetter D, Heinemann S, Liberman MC (1999) Mice lacking $\alpha 9$ acetylcholine receptors have normal vulnerability to acoustic injury. Soc Neurosci Abstr 25:133.

Yoshida N, Hequembourg SJ, Atencio CA, Rosowski JJ, Liberman MC (2000) Acoustic injury in mice: $129 / \mathrm{SvEv}$ is exceptionally resistant to noise-induced hearing loss. Hear Res 141:97-106.

Zuo J, Treadaway J, Buckner TW, Fritzsch B (1999) Visualization of $\alpha 9$ acetylcholine receptor expression in hair cells of transgenic mice containing a modified artificial chromosome. Proc Natl Acad Sci USA 96:14100-14105. 